https://doi.org/10.5194/tc-2021-176

Preprint. Discussion started: 20 July 2021

(c) Author(s) 2021. CC BY 4.0 License.

\title{
A leading-edge based method for correction of slope-induced errors in ice-sheet heights derived from radar altimetry
}

\author{
Weiran Li ${ }^{1}$, Cornelis Slobbe ${ }^{1}$, and Stef Lhermitte ${ }^{1}$ \\ ${ }^{1}$ Department of Geoscience and Remote Sensing, Delft University of Technology, Delft, The Netherlands \\ Correspondence: Weiran Li (w.li-7@tudelft.nl)
}

\begin{abstract}
Satellite radar altimetry has been an important tool for cryospheric applications such as measuring ice-sheet height or assessing snow/ice anomalies (e.g., the extensive melt in Greenland in 2012). Although accurate height measurements are key for such applications, slope-induced errors due to undulating topography within the kilometre-wide pulse-limited footprint can cause multi-meter errors. Therefore different correction methods have been developed ranging from the slope method to

5 the point-based method. Each of these methods have shortcomings as they either neglect the actual topography or the actual footprint that can be estimated by a combination of the leading edge and topography. Therefore, a novel Leading Edge PointBased (LEPTA) method is presented that corrects for the slope-induced error by including the leading edge information of the radar waveform to determine the impact point. The principle of the method is that only the points on the ground that are within range determined by the begin and end of the leading edge are used to determine the impact point.

Benchmarking of the LEPTA method to the slope- and point-based method based on CryoSat-2 LRM acquisitions over Greenland in 2019 shows that heights obtained by LEPTA outperform the other methods when compared to ICESat- 2 observations, both in the flat, interior regions of Greenland and in regions with more complex topography. The median difference between the slope-corrected CryoSat-2 and the ICESat-2 heights is almost negligible, whereas the other methods can have a $0.22 \mathrm{~m}$ and $0.69 \mathrm{~m}$ difference, and the Level-2 data provided by ESA have a $0.01 \mathrm{~m}$ difference. The median absolute deviation, which we use as an indicator of the variation of errors, is also the lowest in LEPTA $(0.09 \mathrm{~m})$ in comparison to the aforementioned methods $(0.22 \mathrm{~m}$ and $0.13 \mathrm{~m})$ and ESA Level-2 data $(0.15 \mathrm{~m})$. Based on that, we recommend considering LEPTA to obtain accurate height measurements with radar altimetry data, especially in regions with complex topography.
\end{abstract}

\section{Introduction}

Satellite radar altimetry is a key tool for assessing the status and dynamics of the cryosphere as it allows constructing digital elevation models (DEMs) (Slater et al., 2018), deriving height change of ice sheets (Helm et al., 2014a), understanding seasonal variations of snow (Adodo et al., 2018), and estimating snowpack properties (Lacroix et al., 2008). To obtain accurate information on heights, altimetry processing involves correction for instrument errors, atmospheric effects, tidal effects, and slope-induced errors (Bouzinac, 2012). Among the correction processes, correction for slope-induced errors has been of crucial importance as they can affect the results of height measurements significantly. For example, according to the error propagation 
https://doi.org/10.5194/tc-2021-176

Preprint. Discussion started: 20 July 2021

(c) Author(s) 2021. CC BY 4.0 License.

(c) (i)

in Brenner et al. (1983), the CryoSat-2 satellite at an altitude of $730 \mathrm{~km}$, and measuring heights of a terrain with a $0.6^{\circ}$ slope, can give a vertical offset of approximately $40 \mathrm{~m}$ and a horizontal offset of $7.6 \mathrm{~km}$.

To correct for the slope-induced errors, different methods have been developed (Brenner et al., 1983; Remy et al., 1989; Bamber, 1994; Roemer et al., 2007). The most widely used methods involve both a correction to the height as well as a relocation of the satellite measurement location from nadir to the expected impact point on the terrain. These correction methods are typically referred to as 'slope' and 'point-based' methods (Levinsen et al., 2016). The slope method assumes constant surface parameters within the altimeter footprint and calculates the relocated longitude, latitude and height according to trigonometry (Brenner et al., 1983; Remy et al., 1989; Bamber, 1994). The point-based method takes the full height information within the satellite footprint and searches for the smallest range from the satellite to the terrain surface (Roemer et al., 2007).

Although both methods have been refined and applied with reliable results, they both show methodological shortcomings.

The slope method, for example, tends to ignore the local topography within the footprint and therefore may not be accurate enough in undulating areas (Levinsen et al., 2016). The point-based method of Roemer et al. (2007), on the other hand, is more accurate in the undulating regions (Roemer et al., 2007; Levinsen et al., 2016) as it considers the detailed topography, but assuming a fixed footprint size neglects the actual footprint that illuminates the terrain. For example, by taking the averaged range within the assumed footprint, this method may ignore part of the terrain that actually contributes to the return signal, or assumes that part of the terrain not visible to the satellite could contribute to the return signal (See Fig. 1). The recent availability of high-resolution DEM products, however, provides the opportunity to determine the part of the terrain contributing to the rise of the leading edge, and therefore helps determining the actual footprint of the radar altimeter.

To overcome the shortcomings of both methods, we present a novel Leading Edge Point-Based (LEPTA) method (Section 3) that exploits high resolution DEM information to correct for the slope-induced error by including the leading edge information of the radar waveform to determine the impact point. The principle of the method is that only the points on the ground that are actually within range determined by the begin and end of the leading edge are used to determine the impact point.

The paper is organised as follows. The data used for radar altimetric processing and result assessment are described. Then, the different methods used for correction and the assessment workflow are introduced. The results and analysis/discussion will then be presented.

\section{Data and pre-processing}

To assess the performance of the LEPTA method, we apply it to all CryoSat-2 LRM acquisitions over Greenland in 2019 and benchmark it to the slope- and point-based methods by comparing it with laser altimeter ICESat- 2 data.

\subsection{CryoSat-2 observations}

On the interior of Greenland ice sheet, data acquired by CryoSat-2 are in Low Resolution Mode (LRM). LRM is the con- 
https://doi.org/10.5194/tc-2021-176

Preprint. Discussion started: 20 July 2021

(c) Author(s) 2021. CC BY 4.0 License.

(c) (i)

$1.65 \mathrm{~km}$ in diameter (Bouzinac, 2012). Our evaluation employs all acquisitions from Jan.-Dec. 2019, resulting in approximately $2.4 \times 10^{6}$ valid acquisitions, in order to ensure abundant spatial and temporal coverage.

To process the waveform information and obtain height estimations, Level-1b (L1b) (European Space Agency, 2019a) waveforms were retracked using the offset centre of gravity (OCOG) method (Wingham et al., 1986) documented in Bamber (1994), because of its precision and robustness (Bamber, 1994; Schröder et al., 2019). According to Davis (1997), a 10\% threshold is ideal for detecting ice-sheet height change, a $20 \%$ threshold is the most proper for estimating the absolute icesheet height, and a 50\% threshold is the most appropriate for estimating the absolute height when the waveform is dominated by surface scattering e.g. Antarctic ice shelves. A 10\% threshold is also applied when volume scattering is strong (Aublanc et al., 2018). In this study, a $20 \%$ threshold is selected to obtain accurate absolute height estimations of the interior of the Greenland ice sheet, as a compromise between surface scattering and volume scattering (Aublanc et al., 2018). The 20\% threshold is close to the $25 \%$ threshold use by ESA L2 processing (Bamber, 1994). This is also a more realistic threshold when the performance is assessed with ICESat-2, which measures the snow-air surface. In addition, in this process, waveforms that do not have a distinguishable noise and beginning of leading edge are dropped.

Additionally, Level-2I (L2I) height data obtained with the OCOG retracker from European Space Agency (2019b) were used as benchmark dataset. In the L2I products the slope-induced error is corrected with the Helm et al. (2014b) DEM, of which the resolution is $1 \mathrm{~km} \times 1 \mathrm{~km}$ (Helm et al., 2014a).

\subsection{ArcticDEM}

Within the slope correction methods, a reference DEM is required to determine the impact point. The slope method therefore uses a low resolution DEM (or a downsampled version) as it assumes a constant slope within the radar footprint. On the contrary, the point-based methods (i.e., LEPTA and the point-based method proposed by (Roemer et al., 2007)) require DEMs with higher resolution, to provide the full information of the local terrain.

In this study, ArcticDEM was used as reference DEM as it is constructed from recent stereo satellite imagery and is available in high resolution $(2 \mathrm{~m} \times 2 \mathrm{~m}$ ) (Porter et al., 2018). The systematic error of ArcticDEM is less than $5 \mathrm{~m}$ (Noh and Howat, 2015) and the DEM has been updated since 2016. ArcticDEM was downsampled to $900 \mathrm{~m}$ resolution for the slope-based method and to $100 \mathrm{~m}$ resolution for the point-based and LEPTA methods used as a compromise between computational efficiency and the demand for high resolution. We also downsample it to various resolutions ( $200 \mathrm{~m}$ to $900 \mathrm{~m}$ with a $100 \mathrm{~m}$ interval) to assess the impact of DEM resolution affects on the correction methods (Subsection 3.3).

\subsection{ICESat-2 observations}

For validation of the different slope correction methods, ICESat-2 L3A Land Ice Height products (Smith et al., 2020a) were used as they provide independent accurate processed height measurements. ICESat-2 uses the Advanced Topographic Laser Altimeter System (ATLAS) which emits green light pulses and counts the received photons (Abdalati et al., 2010). The laser beams are configured in a $2 \times 3$ array. The distance between and within beam pairs is $\sim 3.3 \mathrm{~km}$ and $\sim 90 \mathrm{~m}$, respectively (Smith 
https://doi.org/10.5194/tc-2021-176

Preprint. Discussion started: 20 July 2021

(c) Author(s) 2021. CC BY 4.0 License.

(c) (i)

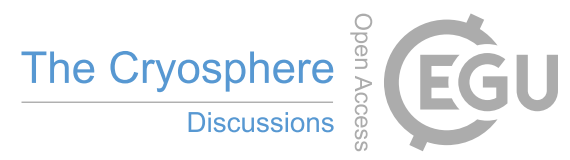

et al., 2019). The along-track resolution of land ice height products is $\sim 20 \mathrm{~m}$ (Smith et al., 2020b). The ICESat-2 data are available at the National Snow and Ice Data Center (NSIDC) website (https://nsidc.org/data/at106).

\section{Methods}

\subsection{Slope correction methods}

The geometry of different slope-induced error correction methods is briefly illustrated in Fig. 1.

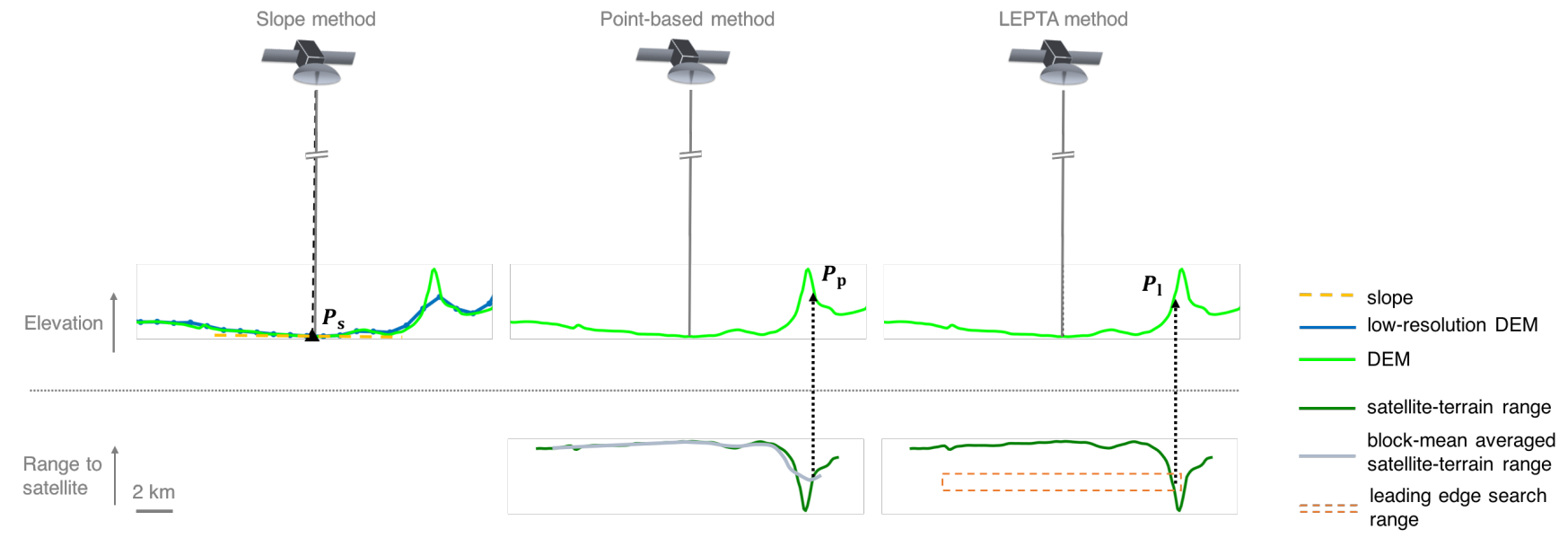

Figure 1. Illustration of different slope-induced error correction methods. The impact points estimated from the slope method, the point-based method, and LEPTA are represented by $P_{s}, P_{p}$ and $P_{l}$. The slope method applies a surface slope based on the DEM, and the point-based method and LEPTA apply the satellite-terrain range, as represented by dark green curves.

\subsubsection{Slope-based correction method}

The slope method uses the slope of the low resolution DEM at the nadir point to compute the impact point. As such it assumes that the slope within the CryoSat-2 footprint is constant, and is defined by direction $\theta$ and magnitude $\Phi$ (Cooper, 1989; Bamber, 1994). The corrected height, represented by $h_{C}$, is the height of the impact point $P_{s}$, can then be obtained by (Bamber, 1994):

$h_{C}=R_{I}-R_{\alpha}=R_{s} \sin (\Phi-\Gamma) / \sin \Phi-R_{\alpha}$

where $\Gamma$ is the central angle between the satellite and $P_{s}, R_{I}$ is the range between $P_{s}$ and the centre of the curvature at $P_{s}$, and $R_{s}$ is the range between the centre of the curvature and the satellite at latitude and longitude $\lambda . R_{\alpha}$ represents the radius of Earth's curvature at $P_{s}$. The corrected location of the impact point in latitude $\phi_{C}$ and longitude $\lambda_{C}$ (in radians) are computed as

$\phi_{C}=\frac{\pi}{2}-2 \sin ^{-1}\left(\frac{X+\Delta x}{2 R_{\alpha} \cos \lambda_{C}}\right)$ 

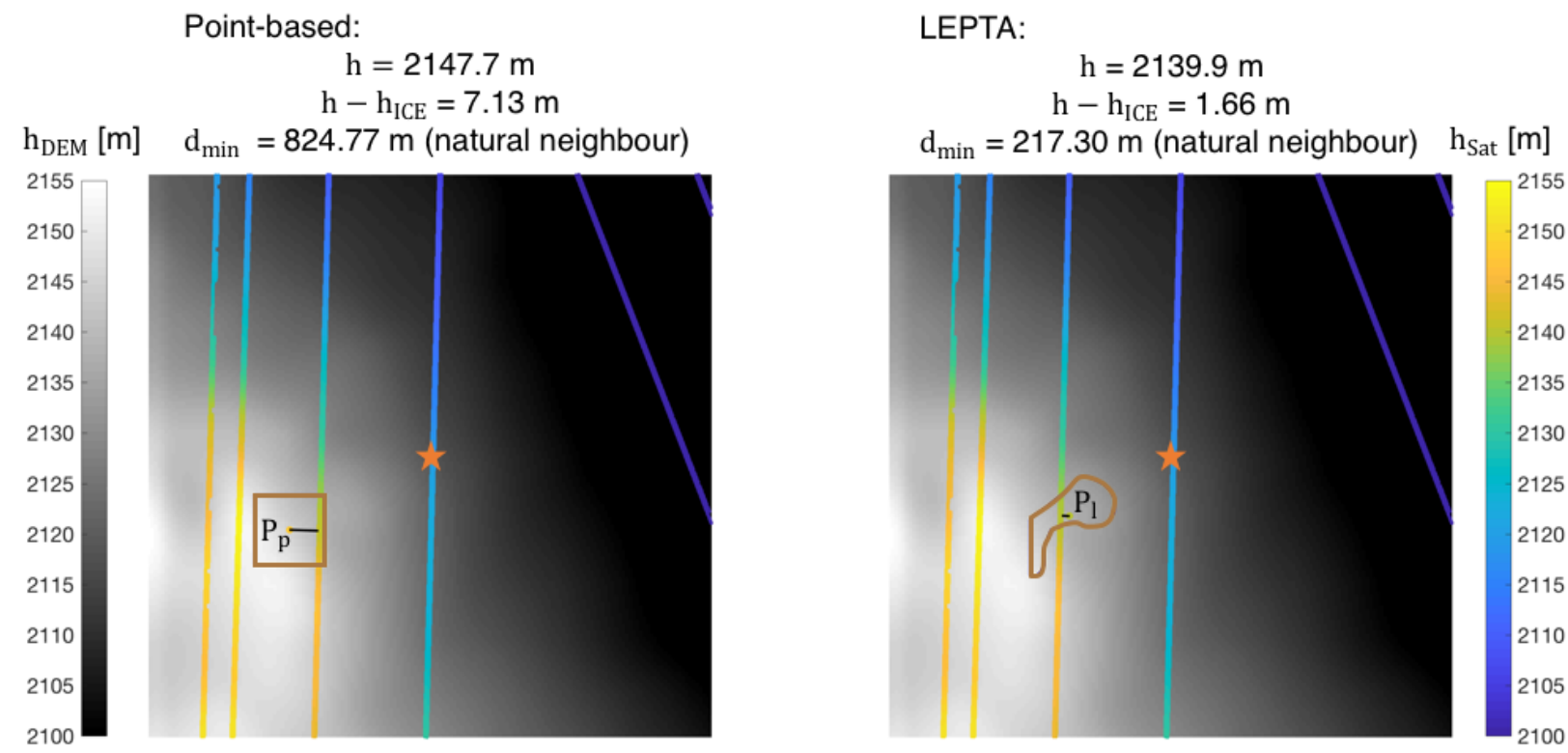

$2 \mathrm{~km}$

Figure 2. Illustration of difference in horizontal geometry of point-based (left) and LEPTA (right) methods. The star marks the nadir point of the satellite measurement, as well as the centre of the region. The brown polygons indicate the assumed CryoSat- 2 footprint. The coloured points at the centre of the polygons (denoted as $P_{p}$ and $P_{l}$ ) are the computed CryoSat-2 impact points, and the coloured points are ICESat-2 measurements. The black line indicates the distance between the corrected CryoSat-2 measurement and the nearest neighbour in ICESat-2 measurements.

$\lambda_{C}=\tan ^{-1}\left(\frac{Y+\Delta y}{X+\Delta x}\right)$

where $X$ and $Y$ define the position of $P_{s}$ in Cartesian coordinates and

$\Delta x=R_{\alpha} \Gamma \cos \theta$

$\Delta y=R_{\alpha} \Gamma \sin \theta$

Application of the slope-method in Fig. 1 shows that the impact point will be assumed at the position $P_{s}$. Inaccuracies usually occur when this method is applied to complex terrains, due to the simplification of the complex topography to a constant slope Levinsen et al. (2016). 
https://doi.org/10.5194/tc-2021-176

Preprint. Discussion started: 20 July 2021

(c) Author(s) 2021. CC BY 4.0 License.

(c) (i)

\subsubsection{Point-based correction method}

The point-based method directly uses the topographic information from the a-priori DEM to find the impact point $\left(P_{p}\right)$. It does so by minimising the mean distance $\bar{R}_{P}$ to the satellite over a pre-defined fixed-size rectangular footprint area (e.g., $2 \mathrm{~km} \times 2$ $\mathrm{km}$ in Roemer et al. (2007)). Assuming the pre-defined rectangular footprint (area $A$ ) consists of $n$ DEM grid cells, $\bar{R}_{P}$ is computed by (Roemer et al., 2007):

$\bar{R}_{P}=\frac{1}{R} \sum_{j=1}^{n} A_{P j} \bar{R}_{P j}$

where $A_{P j}$ and $\bar{R}_{P j}$ are the area and range of each grid cell $j$. The position (defined by $\phi_{c}$ and $\lambda_{c}$ ) of the footprint that minimises $\bar{R}_{P}$ is then obtained as $P_{p}$. The range between the satellite and $P_{p}$ is referred to as $r_{p}$. The corrected height $h_{C}$ is computed as (Roemer et al., 2007)

$h_{C}=h_{N}+r_{p}-\left(h_{S}-h_{I}\right)$,

where $h_{N}$ is the surface height of the nadir point relative to the reference ellipsoid (i.e., $h_{S}$ minus the retracked range), $h_{S}$ is the ellipsoidal height of the satellite, and $h_{I}$ is the DEM height of $P_{p}$. It also shows, however, that this approach can take DEM points into account that actually do not contribute to the rise of the leading edge (i.e., points that fall outside the footprint).

\subsubsection{Leading Edge Point-Based (LEPTA) method}

The LEPTA method is similar to the point-based method as it also uses the topographic information from the a-priori DEM to find the impact point $\left(P_{l}\right)$, but differs in the search method of the impact point. Instead of pre-defining a fixed rectangular pulse-limited footprint size, the LEPTA-method identifies the parts of the terrain that contribute to the rise of the leading edge. To identify these parts, we define the beginning of the leading edge as the point where the normalised waveform power (values are between 0 and 1) is greater than 0.05 . The end of the leading edge is more difficult to define as there might be multiple peaks before the waveform reaches its maximum power. Here, we defined it as the point that is located at a distance $\Delta r$ from the range obtained by applying the OCOG retracker mentioned in Subsection 2.1. In this study, we used $\Delta r=3.5 \mathrm{~m}$. The robustness of the results regarding the choice of $\Delta r$ will be further assessed in Subsection 4.3.

For each point, the distances are computed between the satellite and all DEM grid points within an area of $8 \times 8 \mathrm{~km}$ centred around the nadir point. Thereafter, the DEM points are identified for which the range is within the interval defined by the beginning and end of the leading edge. In case no DEM grid points are identified, the interval is adjusted by the minimum difference between the computed distances and the retracked range. Next, the location of $P_{l}$ is computed as the average of all $K$ identified DEM grid points. Finally, the corrected height $h_{C}$ is computed by

$h_{C}=h_{N}+\sum_{i=1}^{K}\left(r_{\mathrm{DEM}}^{i}-\left(h_{S}-h_{\mathrm{DEM}}^{i}\right)\right)$,

where $h_{\mathrm{DEM}}^{i}$ and $r_{\mathrm{DEM}}^{i}$ are the height of and the range between the satellite and DEM grid point $i$. 
https://doi.org/10.5194/tc-2021-176

Preprint. Discussion started: 20 July 2021

(c) Author(s) 2021. CC BY 4.0 License.

(c) (i)

One of the advantages of the LEPTA method compared to the point-based method is that it includes points that contribute to the rise of the leading edge signal but outside the fixed footprint.

The difference in footprint and impact points between LEPTA-method and point-based method is illustrated in an example in Fig. 2. The brown areas indicate the areas on the surface that (is assumed to) contribute to the return signal for both the point-based and LEPTA method. Contrary to the point-based method, for LEPTA this area may take any shape. This example is an illustration of the theoretical advantage of LEPTA. Assessment of the performance of different methods will be described in Subsection 3.2.

\subsection{Performance assessment}

To assess the performance of the LEPTA method, we benchmark the different methods by comparing their accuracy relative to reference data. First, we directly compare the corrected height measurements for each method with the reference height from the $100 \mathrm{~m}$ ArcticDEM. Doing so provides a conceptual assessment of the performance of the methods. To compare the corrected heights $\left(h_{C}\right)$ with the DEM, we bilinearly interpolate the DEM heights to the CryoSat-2 locations $\left(h_{\text {РЕм }}\right)$. Then, the CryoSat-2 measurements are grouped in $50 \mathrm{~km} \times 50 \mathrm{~km}$ tiles. For each tile, we compute the median and median absolute deviation of the $h_{C}-h_{\mathrm{DEM}}$ values.

Second, we compare the corrected height measurements for each method with the ICESat-2 heights. This comparison is done per month, i.e., we compare the CryoSat-2 heights acquired in a particular month to the ICESat-2 heights acquired in the same month. Also here, we group the datasets in tiles of $50 \mathrm{~km} \times 50 \mathrm{~km}$. For each point, we first identify all ICESat-2 points within $50 \mathrm{~m}$ of the CryoSat-2 point. In case ICESat-2 points are available in each quadrant surrounding the CryoSat-2 point, the ICESat-2 heights are interpolated to the CryoSat-2 point using a natural-neighbour interpolation $\left(h_{\mathrm{ICE} 2}\right)$. Otherwise a nearest neighbour interpolation is applied. A natural neighbour interpolation provides a smoother solution (Bobach, 2009) yet requires weighting functions based on the surrounding points. The differences between the CryoSat- 2 and ICESat- 2 heights are referred to as $\Delta h$. Similar as before, we compute for each tile the median and median absolute deviation of $\Delta h$.

When benchmarking the methods, two aspects of accuracy are assessed. First, we determine the difference between the slopecorrected CryoSat- 2 measurements and the reference heights $\left(h_{\mathrm{DEM}}\right.$ or $h_{\mathrm{ICE} 2}$ ) by means of standard statistical parameters (median, median absolute deviation, mean, and standard deviation). Second, we assess the spatial differences between the methods. The statistical parameters are computed with and without removing outliers. Here, we consider any difference between $h_{C}$ and $h_{\mathrm{DEM}} / h_{\mathrm{ICE} 2}$ outside the 10th and 90th percentiles as an outlier.

\subsection{Sensitivity analysis}

The LEPTA method is potentially sensitive to i) the way the 'end' of the leading edge is defined (which in turn determines the satellite footprint), ii) a bias in the DEM, and iii) the resolution of the used DEM. Another aspect that may impact the results is the adopted OCOG threshold (see Section 2.1). To assess how our choices impact the results, we conducted a number of sensitivity analyses in which we: 
https://doi.org/10.5194/tc-2021-176

Preprint. Discussion started: 20 July 2021

(c) Author(s) 2021. CC BY 4.0 License.

\section{(c) (1)}

- Varied $\Delta r$ from 2-5 $\mathrm{m}$ in steps of $0.5 \mathrm{~m}$.

- Added a bias to the DEM of $-7.5-2.5 \mathrm{~m}$ in steps of $2.5 \mathrm{~m}$.

- Varied the DEM resolution from 200-900 m in steps of $100 \mathrm{~m}$.

- Changed the OCOG threshold from $20 \%$ to $50 \%$.

\section{Results}

\subsection{Comparison with ArcticDEM}

Benchmarking the different methods to the $100 \mathrm{~m}$ ArcticDEM (Table 1) shows that outliers have an important impact on the performance of all methods with multi-metre outliers for the 1st, 5th, 95th and 99th percentiles. We see moreover that the ESA L2I and slope method results include larger outliers than the point-based and LEPTA methods. Since these outliers have a large impact on the mean and standard deviation, we repeated our analysis with all values outside the 10-90th percentile interval removed.

Removing the outliers significantly reduces the standard deviation of $h_{C}-h_{\mathrm{DEM}}$ for all methods and brings the mean closer to the median. Comparison of the mean and median values (Table 1) and probability distribution (Fig. 3) indicates moreover that LEPTA performs best when compared with ArcticDEM, with both mean and median differences in height of $0.27 \mathrm{~m}$. The slope method gives the largest mean difference of $1.08 \mathrm{~m}$, while the point-based method gives the largest median of $0.95 \mathrm{~m}$. The standard deviation $(0.44 \mathrm{~m})$ and median absolute deviation $(0.33 \mathrm{~m})$ of LEPTA are also the smallest. The largest deviation values after outlier removal are given by the slope method, with the standard deviation being $1.09 \mathrm{~m}$ and median absolute deviation being $0.50 \mathrm{~m}$. This shows that the $h_{C}-h_{\mathrm{DEM}}$ results from the slope method are the least represented by the mean and median, thus are the least ideal. An additional note is that the mean and median from all methods become above 0 , which means that the heights obtained by these methods are generally higher than ArcticDEM heights. It is also noticed that the ESA L2I products result in more valid outputs than the self-implemented methods.

Comparison of the spatial patterns of median and median absolute deviation (Fig. 3) shows large spatial differences in both pattern and magnitude between the different methods. In general, the largest median and median absolute deviation values occur closer to the margins of Greenland, where the terrain is steeper. For ESA L2I, the point-based method and LEPTA, the median values on the eastern side of the ice sheet are generally the highest. The median absolute deviation values from ESA L2I are largest on the western side of the ice sheet. For the slope method, the largest median and median absolute deviation values occur on both eastern and western sides of Greenland, and largely exceed those of the ESA L2I products, the point-based method, and LEPTA. In addition, although hard to distinguish from the plots, $54.2 \%$ of the grid-cells from the point-based method have higher median absolute deviation than LEPTA. High median absolute deviation values from the point-based method exist, but are not so concentrated close to Jakobshavn as the other methods. Combining the statistics in Table 1 and 
https://doi.org/10.5194/tc-2021-176

Preprint. Discussion started: 20 July 2021

(C) Author(s) 2021. CC BY 4.0 License.

Table 1. Statistics of the height difference between slope-corrected CryoSat-2 measurements and ArcticDEM and ICESat-2. Height statistics are in unit of metres. Before and after in the table represent the parameters before and after removing outliers (referred to as w/ outlier and w/o outlier) using 10th and 90th percentiles. E, S, P and L represent ESA L2I, slope method, point-based method and LEPTA, respectively.

\begin{tabular}{|c|c|c|c|c|c|c|c|c|c|}
\hline \multirow{2}{*}{\multicolumn{2}{|c|}{ CryoSat-2 }} & \multicolumn{4}{|c|}{ vs. ArcticDEM } & \multicolumn{4}{|c|}{ vs. ICESat-2 } \\
\hline & & \multirow{3}{*}{$\begin{array}{l}\mathrm{E} \\
2.4 \mathrm{e} 6 \\
2.0 \mathrm{e} 6\end{array}$} & \multirow{3}{*}{$\begin{array}{l}\mathrm{S} \\
2.2 \mathrm{e} 6 \\
1.8 \mathrm{e} 6\end{array}$} & \multirow{3}{*}{$\begin{array}{l}\mathrm{P} \\
2.2 \mathrm{e} 6 \\
1.8 \mathrm{e} 6\end{array}$} & \multirow{3}{*}{$\begin{array}{l}\mathrm{L} \\
2.2 \mathrm{e} 6 \\
1.8 \mathrm{e} 6\end{array}$} & \multirow{3}{*}{$\begin{array}{l}\mathrm{E} \\
9.1 \mathrm{e} 4 \\
7.3 \mathrm{e} 4\end{array}$} & \multirow{3}{*}{$\begin{array}{l}\mathrm{S} \\
8.3 \mathrm{e} 4 \\
6.6 \mathrm{e} 4\end{array}$} & \multirow{3}{*}{$\begin{array}{l}\mathrm{P} \\
8.3 \mathrm{e} 4 \\
6.6 \mathrm{e} 4\end{array}$} & \multirow{3}{*}{$\begin{array}{l}\mathrm{L} \\
8.2 \mathrm{e} 4 \\
6.6 \mathrm{e} 4\end{array}$} \\
\hline & w/ outlier & & & & & & & & \\
\hline & w/o outlier & & & & & & & & \\
\hline \multirow{2}{*}{ 1st percentile } & w/ outlier & -41.7 & -1.18 & -4.37 & -2.43 & -39.7 & -0.51 & -2.15 & -1.12 \\
\hline & w/o outlier & -0.85 & -0.17 & 0.03 & -0.60 & -0.37 & -0.08 & 0.42 & -0.25 \\
\hline \multirow{2}{*}{ 5th percentile } & w/ outlier & -3.13 & -0.48 & -0.52 & -1.03 & -2.00 & -0.17 & 0.28 & -0.42 \\
\hline & w/o outlier & -0.61 & -0.06 & 0.18 & -0.46 & -0.28 & -0.05 & 0.46 & -0.20 \\
\hline \multirow{2}{*}{ 10th percentile } & w/ outlier & -0.94 & -0.20 & -0.01 & -0.64 & -0.40 & -0.09 & 0.40 & -0.26 \\
\hline & w/o outlier & -0.42 & 0.06 & 0.32 & -0.34 & -0.22 & -0.02 & 0.49 & -0.16 \\
\hline \multirow{2}{*}{ 90th percentile } & w/ outlier & 2.02 & 5.12 & 1.90 & 1.22 & 1.62 & 4.80 & 1.31 & 0.44 \\
\hline & w/o outlier & 1.27 & 2.65 & 1.58 & 0.88 & 0.68 & 2.21 & 1.06 & 0.20 \\
\hline \multirow{2}{*}{ 95th percentle } & w/ outlier & 3.38 & 9.23 & 2.25 & 1.75 & 3.41 & 9.09 & 1.64 & 1.19 \\
\hline & w/o outlier & 1.55 & 3.56 & 1.72 & 1.02 & 1.01 & 3.20 & 1.17 & 0.27 \\
\hline \multirow{2}{*}{ 99th percentile } & w/ outlier & 10.06 & 23.62 & 2.97 & 4.63 & 11.12 & 22.72 & 2.44 & 5.16 \\
\hline & w/o outlier & 1.90 & 4.74 & 1.86 & 1.17 & 1.48 & 4.42 & 1.28 & 0.39 \\
\hline \multirow{2}{*}{ median } & w/ outlier & 0.36 & 0.75 & 0.95 & 0.27 & 0.01 & 0.22 & 0.69 & 0.00 \\
\hline & w/o outlier & 0.36 & 0.75 & 0.95 & 0.27 & 0.01 & 0.22 & 0.69 & 0.00 \\
\hline \multirow{2}{*}{$\begin{array}{l}\text { median absolute } \\
\text { deviation }\end{array}$} & w/ outlier & 0.57 & 0.66 & 0.46 & 0.44 & 0.21 & 0.28 & 0.18 & 0.12 \\
\hline & w/o outlier & 0.43 & 0.50 & 0.35 & 0.33 & 0.15 & 0.22 & 0.13 & 0.09 \\
\hline \multirow{2}{*}{ mean } & w/ outlier & -0.73 & 2.09 & 0.76 & 0.32 & -0.82 & 1.78 & 0.67 & 0.15 \\
\hline & w/o outlier & 0.39 & 1.08 & 0.95 & 0.27 & 0.12 & 0.67 & 0.74 & 0.01 \\
\hline \multirow{2}{*}{ standard deviation } & w/ outlier & 8.39 & 5.76 & 2.68 & 1.56 & 10.07 & 5.86 & 1.69 & 2.02 \\
\hline & w/o outlier & 0.63 & 1.09 & 0.46 & 0.44 & 0.38 & 1.03 & 0.21 & 0.14 \\
\hline
\end{tabular}

the spatial distribution of median and median absolute deviation in Fig. 3, it can be concluded that LEPTA has the most ideal performance, when compared with ArcticDEM.

\subsection{Validation with ICESat-2 observations}

Comparison of CryoSat-2 and ICESat-2 heights (Fig. 4) shows again the importance of outliers on the slope-correction methods although the outliers are typically lower than for the ArcticDEM comparison. For all methods, the $\Delta h$ values within the visualised range in the probability distribution plot have a longer tail on the right side than the left side of the median. Very large outliers (1st and 99th percentiles) occur at $-39.7 \mathrm{~m}$ for ESA L2I and at $22.72 \mathrm{~m}$ for the slope method. 
https://doi.org/10.5194/tc-2021-176

Preprint. Discussion started: 20 July 2021

(c) Author(s) 2021. CC BY 4.0 License.
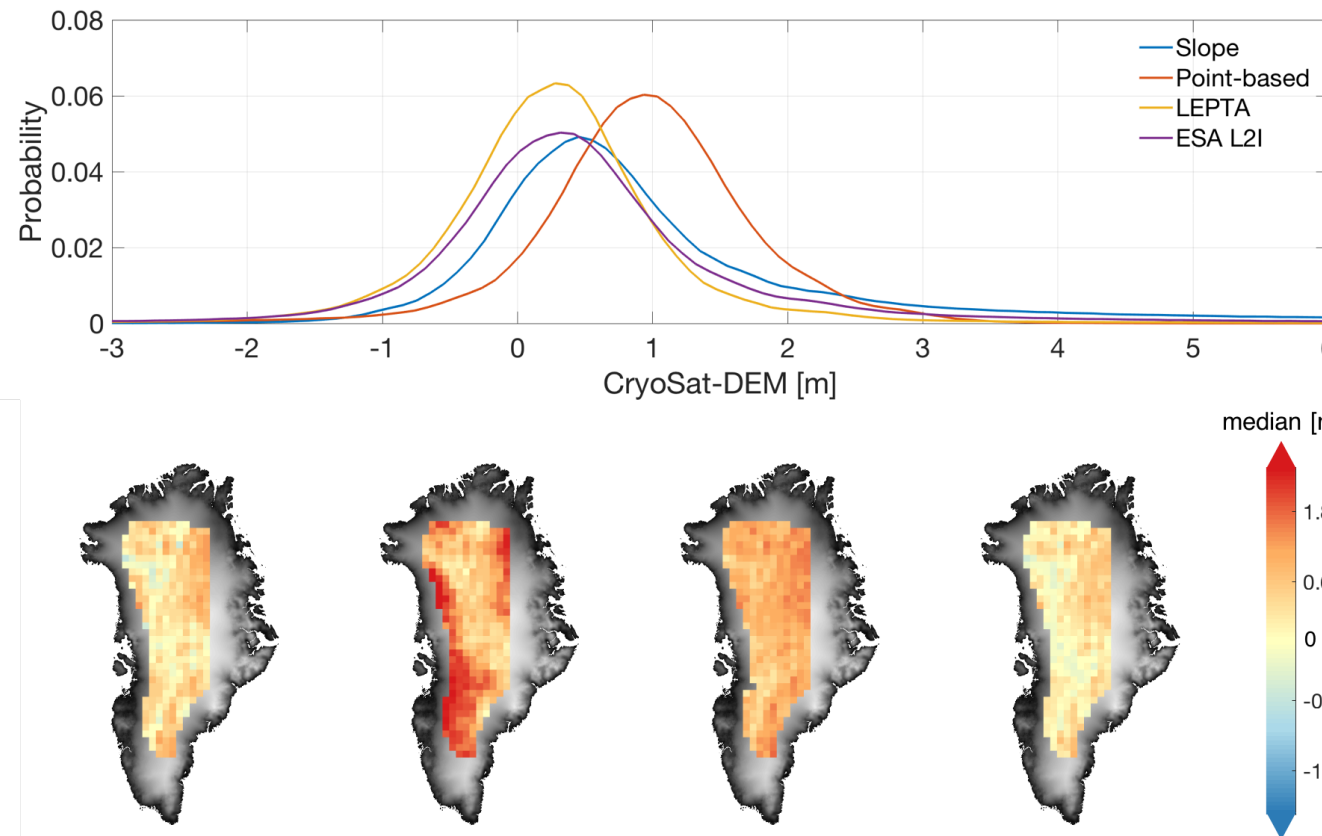

median [m] 
https://doi.org/10.5194/tc-2021-176

Preprint. Discussion started: 20 July 2021

(c) Author(s) 2021. CC BY 4.0 License.
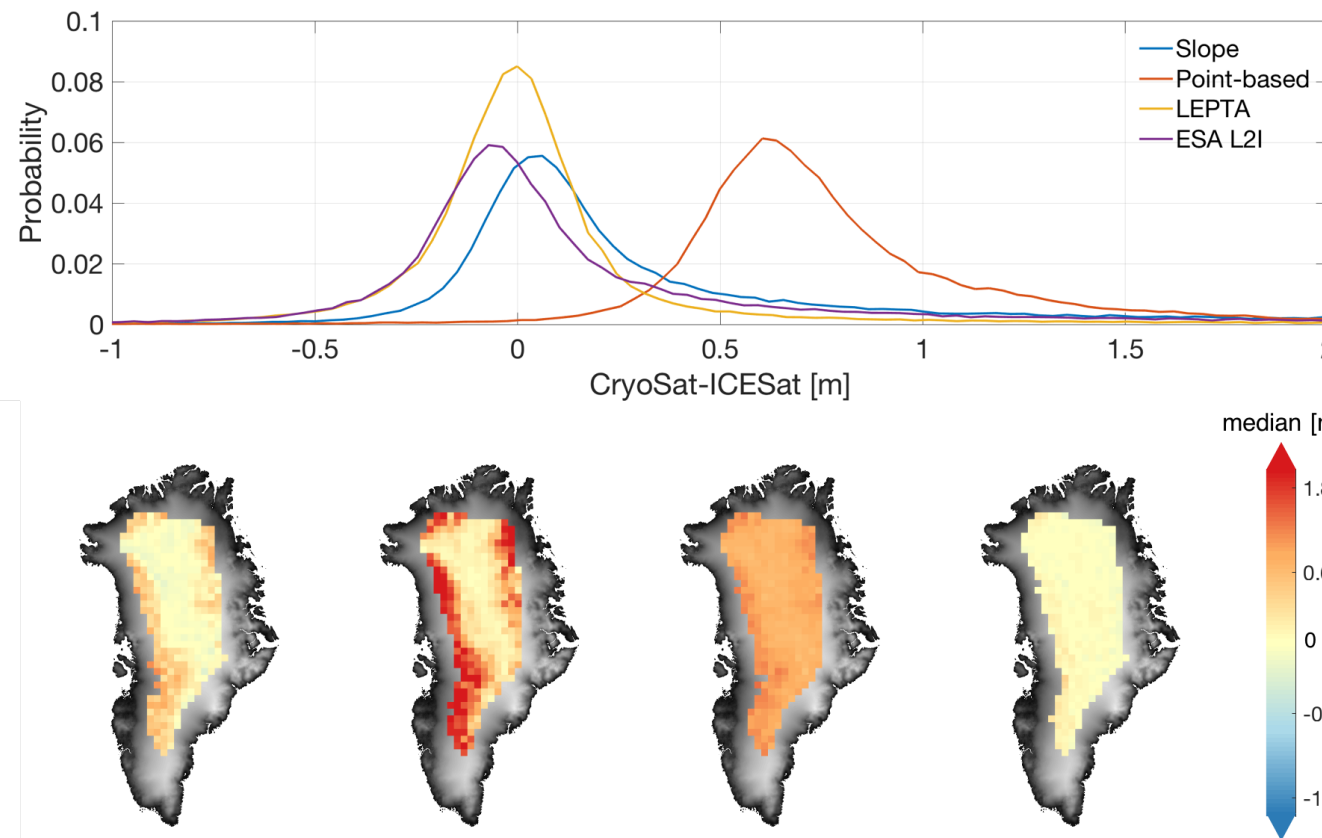

median [m]

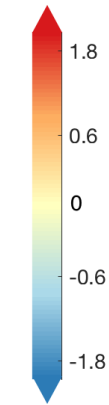

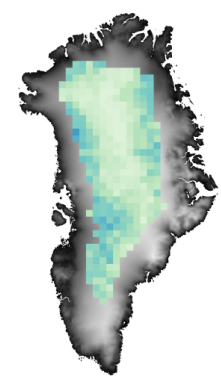

ESA L2I

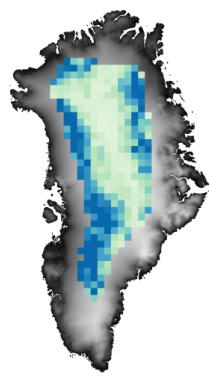

Slope

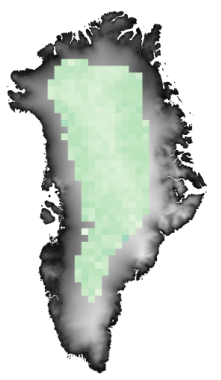

Point-based

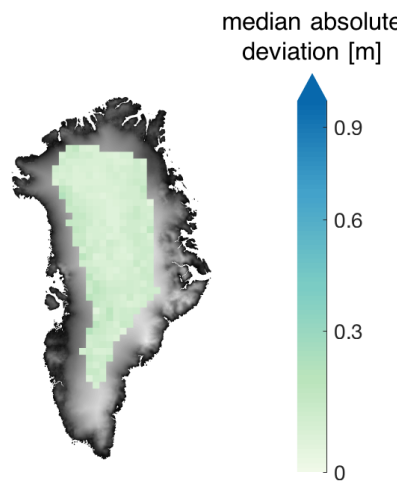

LEPTA

Figure 4. Upper panel: probability distribution of height difference between CryoSat-2 and the ICESat-2, before removing the outliers, and medium and lower panel: spatial distribution of median and median absolute deviation of the height difference per tile of $50 \mathrm{~km} \times 50 \mathrm{~km}$, after removing the outliers. The probability distribution is plotted with all data samples, but restricted to [-1 $\mathrm{m}, 2 \mathrm{~m}]$ for visualisation. The colours of the gridded plots are in logarithmic scale. The spatial distribution results from left to right are obtained by ESA L2I products, the slope method, the point-based method and LEPTA, with the $1 \mathrm{~km} \times 1 \mathrm{~km}$ DEM covering Greenland (Helm et al., 2014a, b) as background.

With the outliers removed, the standard deviation of $\Delta h$ values from all methods is greatly reduced, especially for the ESA L2I and slope-method which show the largest outliers. The lowest median $(0.00 \mathrm{~m})$, mean $(0.01 \mathrm{~m})$, median absolute deviation 
https://doi.org/10.5194/tc-2021-176

Preprint. Discussion started: 20 July 2021

(c) Author(s) 2021. CC BY 4.0 License.

(c) (i)

$(0.09 \mathrm{~m})$ and standard deviation $(0.14 \mathrm{~m})$ of $\Delta h$ are obtained by LEPTA, showing that the LEPTA method again outperforms the other methods. The largest median $(0.69 \mathrm{~m})$ and mean $(0.74 \mathrm{~m})$ are obtained by the point-based method, and the largest median absolute deviation $(0.22 \mathrm{~m})$ and standard deviation $(1.03 \mathrm{~m})$ are from the slope method. This indicates that the heights obtained by LEPTA are the closest to the ICESat- 2 heights.

Comparison of the height differences between CryoSat-2 and ArcticDEM and ICESat-2, respectively, shows moreover that the height differences with ICESat-2 are smaller, probably due to the longer time gap between CryoSat-2 and ArcticDEM, as satellite imagery data for generating ArcticDEM were gathered since 2007 (Noh and Howat, 2017; Howat et al., 2019), whereas ICESat-2 measurements were obtained in the same month as CryoSat-2 data.

The spatial distribution of the mean and median absolute deviation of $\Delta h$ relative to ICESat-2 (Fig. 4) shows clear spatial patterns for $\Delta h$, where the LEPTA method again outperforms the other methods with lower height differences compared to the point-based method (i.e. median values of 0 versus $0.69 \mathrm{~m}$, respectively) and more spatially homogeneous patterns compared to ESA's L2I products and the slope method. The latter show for example large errors over the steeper areas near the edges. To sum up, the point-based method generally has the highest median values, while the slope method has the largest contrast between the interior and the margins.

\subsection{Sensitivity to the definition of the end of the leading edge}
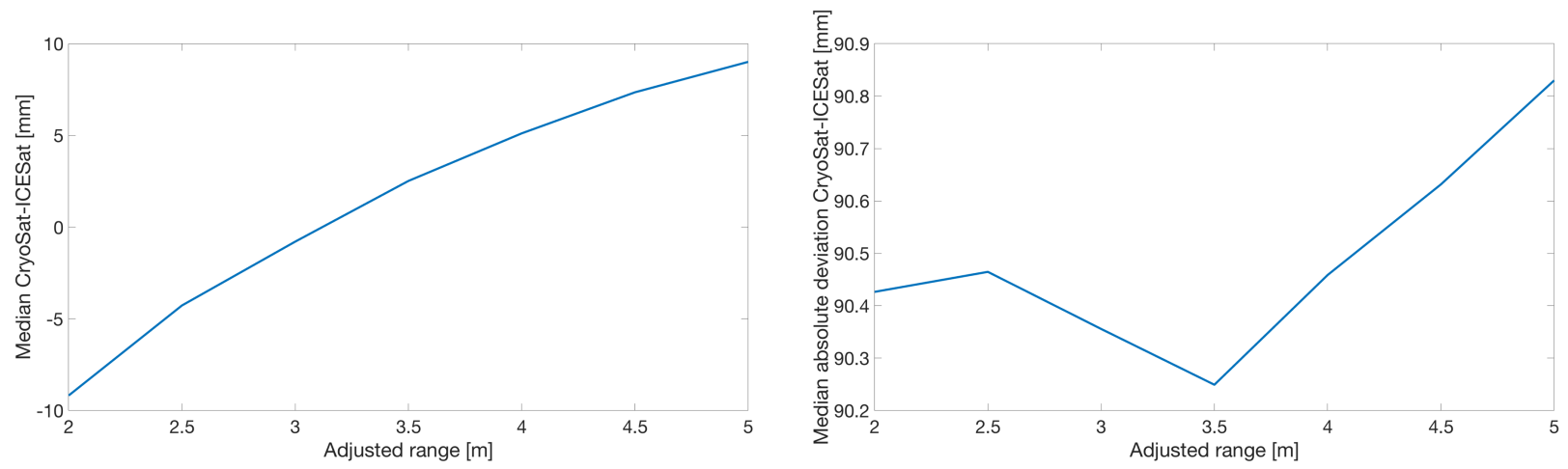

Figure 5. Median and median absolute deviation as function of $\Delta r$. As $\Delta r$ increases, the former curve varies within millimetre level, and the latter varies within centimetre level.

As mentioned in Subsection 3.3, the performance of the LEPTA method relies on the definition of the beginning and end of the leading edge, where the definition of the end of the leading edge is particularly difficult. To assess the sensitivity of LEPTA by the choice of $\Delta r$, we repeated the performance assessment by defining the end of the leading edge as the point which is 2-5 m away from the retracking point. The results are summarised in Fig. 5. It shows that, while $\Delta r$ changes at metre level, the median values of $\Delta h$ change at millimetre level, and the median absolute deviation values change at centimetre level. This analysis confirms that the method is very robust to the choice of $\Delta r$. An additional observation is that the slope of the curve 
https://doi.org/10.5194/tc-2021-176

Preprint. Discussion started: 20 July 2021

(c) Author(s) 2021. CC BY 4.0 License.

(c) (i)

of median values decreases with increasing $\Delta r$. A reason for this could be that when the range interval is large enough, $1 \mathrm{~m}$ of increase does not necessarily increase the amount of the illuminated points on the terrain.

\subsection{Sensitivity to biases in the DEM}
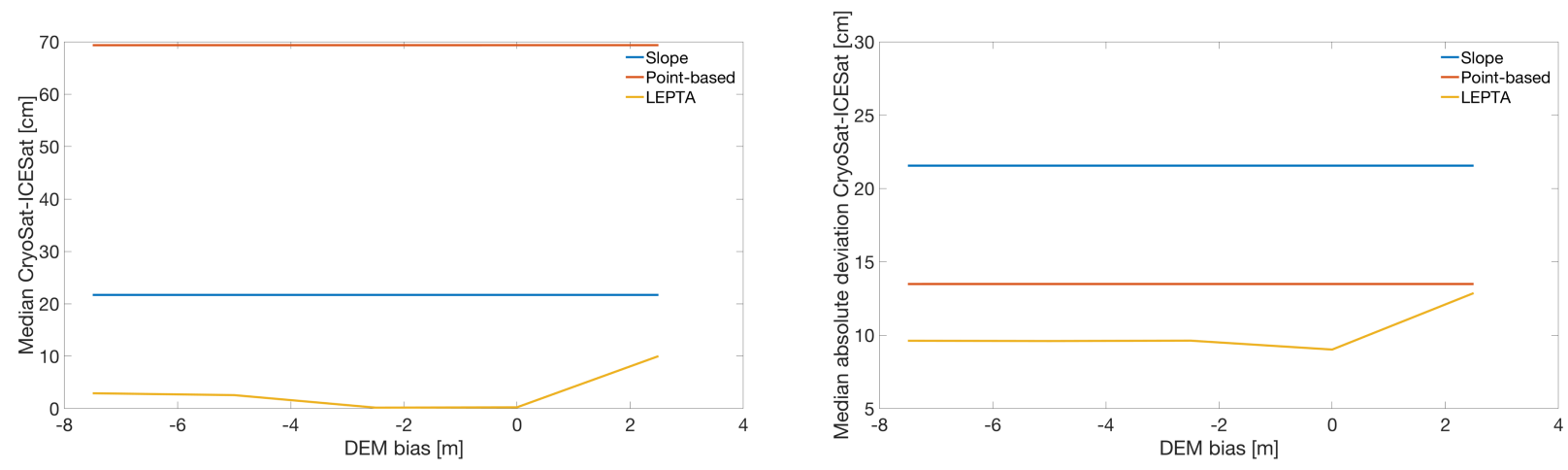

Figure 6. Median and median absolute deviation as function of a bias in the DEM. LEPTA performs best with the original DEM.

To assess the sensitivity of the methods to biases in the DEM (e.g. due to surface mass balance changes), we performed the analysis introduced in Subsection 3.3 with ICESat-2 as validation data. Figure 6 shows that the slope and the point-based methods are not affected by biases in the DEM, while for LEPTA the median changes about $10 \mathrm{~cm}$ when the bias in the DEM is $2.5 \mathrm{~m}$. The median absolute deviation is also around $3 \mathrm{~cm}$ higher. This is understandable as the presence of a bias in the DEM does not affect the slope or the relative differences between the DEM points, which are the key information to the slope method and the point-based method respectively. However, in the case of LEPTA, when the DEM heights are biased, the search range is different from using the heights of the original DEM heights. Using the same search range may ignore some points on the terrain that actually contribute to the waveform leading edge, resulting in a slightly larger bias. This result indicates that LEPTA is relatively sensitive to the bias in DEM heights, compared to other methods.

\subsection{Sensitivity to the resolution of the DEM}

Figure 7 shows the effect of changing the DEM resolution on the median and median absolute deviation. The point-based method has the largest deviation when the DEM resolution changes from $200 \mathrm{~m}$ to $900 \mathrm{~m}$. Although not directly visible in the plots, the median of the slope method changes from $0.29 \mathrm{~m}$ to $0.22 \mathrm{~m}$, and that of LEPTA changes from $0.00 \mathrm{~m}$ to 0.04 $\mathrm{m}$. The median absolute deviation of LEPTA changes from $0.09 \mathrm{~m}$ to $0.10 \mathrm{~m}$. The sensitivity analysis of the DEM resolution again shows the robustness of LEPTA method, as well as the importance of accurate high-resolution DEM information for the point-based method. This is reversed for the slope method where coarser DEM resolutions result in better performance as the overall slope is better represented. This simple assessment confirms that for the point-based method and LEPTA, the height difference from ICESat-2 is small when corrected with a high-resolution DEM, and those from the slope method are small when corrected with a low-resolution DEM. 

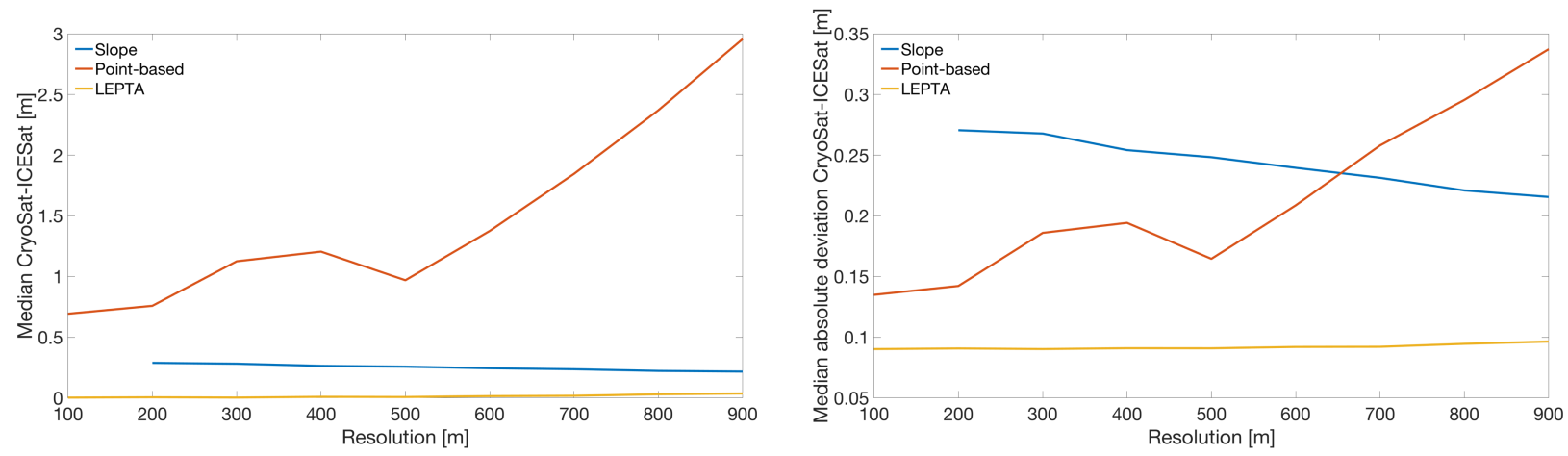

Figure 7. Median and median absolute deviation as function of DEM resolution. Both the point-based method and LEPTA have a decreasing accuracy with decreasing DEM resolution, while the slope method has an increasing accuracy with an decreasing DEM resolution.

\subsection{Assessment of retracker dependence}

Table 2. Statistics of the height difference between slope-corrected CryoSat-2 measurements and ICESat-2, with $20 \%$ and $50 \%$ threshold for the applied OCOG retracker. Height statistics are in unit of metres. Outliers are removed using 10th and 90th percentiles. S, P, and L represent the slope method, point-based method, and LEPTA, respectively.

\begin{tabular}{|l|lll|lll|}
\hline \multirow{2}{*}{ CryoSat-2 } & \multicolumn{3}{|c|}{$20 \%$ threshold } & \multicolumn{3}{c|}{$50 \%$ threshold } \\
& $\mathrm{S}$ & $\mathrm{P}$ & $\mathrm{L}$ & $\mathrm{S}$ & $\mathrm{P}$ & $\mathrm{L}$ \\
\hline median & 0.22 & 0.69 & 0.00 & -0.11 & 0.30 & -0.33 \\
\hline median absolute deviation & 0.21 & 0.13 & 0.09 & 0.21 & 0.14 & 0.14 \\
\hline mean & 0.67 & 0.74 & 0.01 & 0.18 & 0.27 & -0.38 \\
\hline standard deviation & 1.03 & 0.21 & 0.14 & 0.76 & 0.25 & 0.24 \\
\hline
\end{tabular}

Comparison of the different slope-correction methods with the ESA L2I products highlights the importance of the retracker in the different height estimations. Changing the OCOG threshold from $20 \%$ to $50 \%$ results in retracked points located further away from the satellite, lowering the height estimations (Table 2). For all methods, this expected behaviour is apparent, as the median of the height difference between CryoSat-2 and ICESat-2 reduces with $30 \mathrm{~cm}$ and $40 \mathrm{~cm}$ compared to the original threshold. The median absolute deviation increases, showing that the height estimations from the $50 \%$ retracker are slightly more off. This result indicates that the height estimations from LEPTA are dependent on the choice of retracker, which is the same as the other methods. 
https://doi.org/10.5194/tc-2021-176

Preprint. Discussion started: 20 July 2021

(c) Author(s) 2021. CC BY 4.0 License.

(c) (i)

\section{Discussion}

The combination of validation based on ArcticDEM and ICESat-2 shows that the presented LEPTA method outperforms the slope and point-based methods in accuracy with lower median, mean, and median absolute deviations from the reference datasets. Also in terms of spatial patterns, the LEPTA method outperforms the other methods, especially compared to the slope based method that shows large error in the steeper margin regions. This indicates that including leading edge information to determine the impact point has an important improvement on the accuracy of CryoSat-2 height estimations. Our results show moreover that the method is not very sensitive to changes in the definition of the end of the leading edge as it shows only millimetre-level uncertainties for the corrected heights when including multi-metre uncertainties on the definition of the end of the leading edge. However, the definition of the leading edge should be adjusted accordingly when a bias is introduced in the DEM, for example as a result long term height changes due surface mass balance changes since the DEM construction. The DEM resolution also has little impact on the method, although a high-resolution DEM is recommended. On the contrary, the choice of the retracker affects all the methods to a similar extent. This analysis also agrees with the study of Brenner et al. (2007) that the difference between radar and laser altimeters is retracker-dependent.

By showing the importance of impact points over steeper margin areas, our results confirm earlier work of Levinsen et al. (2016) in the margin regions, where they also showed that the point-based method outperforms the slope based methods. The improved performance of the point-based method and LEPTA methods can be explained by assumption of a constant slope within the footprint in the slope-based method, which results in a biased impact point further away from the satellite than the optimal location (Levinsen et al., 2016). An explanation for this improved performance of LEPTA over the point-based method can be found in the design of the method which only takes into account areas that are within the footprint of CryoSat-2 (Fig. 1 and Fig. 2).

Comparison of the ESA L2 products with the slope-based correction method shows finally that the ESA product outperforms standard slope correction method. This agrees with Levinsen et al. (2016) who attributed the different performance between the ESA L2 products and the self-implementation to the Doppler slope correction implemented in ESA L2 products (Blarel and Legresy, 2012) and DEM differences. The ESA L2 products also provides more valid outputs, compared to the selfimplemented methods. This could also be attributed to the Doppler slope correction as it has the advantage of being valid directly for all continental surfaces (Blarel and Legresy, 2012). On the contrary, the self-implemented methods dropped the waveforms that are not similar to the standardised waveform (as in Fig. 1 of Simonsen and Sørensen (2017)). LEPTA also leaves out the points that do not have valid DEM values within the search range. More invalid points are therefore dropped in this process. This phenomenon leaves LEPTA potential for improvement.

\section{Conclusions}

Reducing slope-induced errors is a key correction algorithm when applying the radar altimeter data. To correct for this error, different methods have been developed to determine the impact point, which all rely on footprint assumptions: e.g. slopemethod, which assumes a constant slope within the footprint, or the point-based method, which assumes a fixed footprint size 
https://doi.org/10.5194/tc-2021-176

Preprint. Discussion started: 20 July 2021

(c) Author(s) 2021. CC BY 4.0 License.

(c) (i)

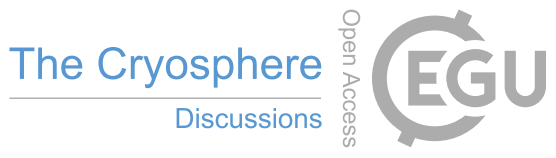

and defines the reflecting point as the shortest mean range of points within each assumed footprint. Each of these methods has shortcoming as they either neglect the actual topography or the actual footprint that can be estimated by a combination of the leading edge and topography.

To overcome this shortcoming, we present a novel Leading Edge Point-Based (LEPTA) method that corrects for the slopeinduced error by including the leading edge information of the radar waveform to determine the impact point. The principle of the method is that only the points on the ground that are within range determined by the begin and end of the leading edge are used to determine the impact point. This requires the assistance of a high-resolution DEM, e.g. $100 \mathrm{~m}$ resolution.

Statistics show that the LEPTA method outperforms all other methods with the smallest median and variability of errors. The median is almost identical to the ICESat-2 height measurements. Spatially, LEPTA has a good improvement compared to the traditional slope method on the margins of the ice sheet. Therefore, LEPTA is a method worth considering to obtain accurate height measurements with radar altimeter, especially in regions with complex topography.

Author contributions. WL conducted data management, processing and analysis, produced the figures and provided the manuscript with contributions from all co-authors. CS designed the study and provided expertise and software for radar-altimetry processing. SL provided support on statistical analysis and data visualisation.

Acknowledgements. The research is supported by the Dutch Research Council (NWO) on the ALWGO.2017.033 project.

ArcticDEM is provided by the Polar Geospatial Center under NSF-OPP awards 1043681, 1559691, and 1542736. DEM of Greenland used for result visualisation is provided by Helm et al. (2014a, b) under license Creative Commons Attribution 3.0 Unported. The CryoSat-2 L1b and L2I data are provided online by ESA and the ICESat-2 L3A data are provided online by NSIDC.

315 The authors would also like to thank Professor Roland Klees, Dr Bert Wouters, Katarzyna Sejan, Jan Haacker and Dr Lorenzo Iannini for valuable discussions, and Dr Louise Sandberg Sørensen for the review and editing of this draft. 
https://doi.org/10.5194/tc-2021-176

Preprint. Discussion started: 20 July 2021

(c) Author(s) 2021. CC BY 4.0 License.

\section{References}

Abdalati, W., Zwally, H. J., Bindschadler, R., Csatho, B., Farrell, S. L., Fricker, H. A., Harding, D., Kwok, R., Lefsky, M., Markus, T., et al.: The ICESat-2 laser altimetry mission, Proceedings of the IEEE, 98, 735-751, 2010.

Adodo, F. I., Remy, F., and Picard, G.: Seasonal variations of the backscattering coefficient measured by radar altimeters over the Antarctic Ice Sheet, The Cryosphere, 12, 1767-1778, https://doi.org/10.5194/tc-12-1767-2018, 2018.

Aublanc, J., Moreau, T., Thibaut, P., Boy, F., Rémy, F., and Picot, N.: Evaluation of SAR altimetry over the antarctic ice sheet from CryoSat-2 acquisitions, Advances in Space Research, 62, 1307-1323, https://doi.org/10.1016/j.asr.2018.06.043, 2018.

Bamber, J. L.: Ice sheet altimeter processing scheme, International Journal of Remote Sensing, 15, 925-938, https://doi.org/10.1080/01431169408954125, 1994.

Blarel, F. and Legresy, B.: Investigations on the Envisat RA2 Doppler slope correction for ice sheets, in: European Space Agency-CNES Symp., Venice, Italy, 2012.

Bobach, T. A.: Natural Neighbor Interpolation - Critical Assessment and New Contributions, Ph.D. thesis, Technische Universität Kaiserslautern, 2009.

Bouzinac, C.: CryoSat Product Handbook, Tech. rep., ESA, https://earth.esa.int/documents/10174/125272/CryoSat_Product_Handbook, 2012.

Brenner, A. C., Bindschadler, R. A., Thomas, R. H., and Zwally, H. J.: Slope-induced errors in radar altimetry over continental ice sheets, Journal of Geophysical Research, 88, 1617, https://doi.org/10.1029/jc088ic03p01617, 1983.

Brenner, A. C., DiMarzio, J. P., and Zwally, H. J.: Precision and Accuracy of Satellite Radar and Laser Altimeter Data Over the Continental Ice Sheets, IEEE Transactions on Geoscience and Remote Sensing, 45, 321-331, https://doi.org/10.1109/tgrs.2006.887172, 2007.

Cooper, A.: Slope Correction By Relocation For Satellite Radar Altimetry, in: 12th Canadian Symposium on Remote Sensing Geoscience and Remote Sensing Symposium, IEEE, https://doi.org/10.1109/igarss.1989.577978, 1989.

Davis, C.: A robust threshold retracking algorithm for measuring ice-sheet surface elevation change from satellite radar altimeters, IEEE Transactions on Geoscience and Remote Sensing, 35, 974-979, https://doi.org/10.1109/36.602540, 1997.

European Space Agency: L1b LRM Precise Orbit. Baseline D, https://doi.org/10.5270/CR2-cbow23i, 2019a.

European Space Agency: L2 LRM Precise Orbit. Baseline D, https://doi.org/10.5270/CR2-k1o4pyh, $2019 \mathrm{~b}$.

Helm, V., Humbert, A., and Miller, H.: Elevation and elevation change of Greenland and Antarctica derived from CryoSat-2, The Cryosphere, 8, 1539-1559, https://doi.org/10.5194/tc-8-1539-2014, 2014a.

Helm, V., Humbert, A., and Miller, H.: Elevation Model of Greenland derived from CryoSat-2 in the period 2011 to 2013 , links to DEM and uncertainty map as GeoTIFF, https://doi.org/10.1594/PANGAEA.831393, 2014b.

Howat, I. M., Porter, C., Smith, B. E., Noh, M.-J., and Morin, P.: The Reference Elevation Model of Antarctica, The Cryosphere, 13, 665-674, https://doi.org/10.5194/tc-13-665-2019, 2019.

Lacroix, P., Dechambre, M., Legrésy, B., Blarel, F., and Rémy, F.: On the use of the dual-frequency ENVISAT altimeter to determine snowpack properties of the Antarctic ice sheet, Remote Sensing of Environment, 112, 1712-1729, https://doi.org/10.1016/j.rse.2007.08.022, 2008.

Levinsen, J. F., Simonsen, S. B., Sorensen, L. S., and Forsberg, R.: The Impact of DEM Resolution on Relocating Radar Altimetry Data Over Ice Sheets, IEEE Journal of Selected Topics in Applied Earth Observations and Remote Sensing, 9, 3158-3163, https://doi.org/10.1109/jstars.2016.2587684, 2016. 
https://doi.org/10.5194/tc-2021-176

Preprint. Discussion started: 20 July 2021

(c) Author(s) 2021. CC BY 4.0 License.

(c) (i)

Noh, M.-J. and Howat, I. M.: Automated stereo-photogrammetric DEM generation at high latitudes: Surface Extraction with TIN-based Search-space Minimization (SETSM) validation and demonstration over glaciated regions, GIScience \& Remote Sensing, 52, 198-217, https://doi.org/10.1080/15481603.2015.1008621, 2015.

Noh, M.-J. and Howat, I. M.: The Surface Extraction from TIN based Search-space Minimization (SETSM) algorithm, ISPRS Journal of Photogrammetry and Remote Sensing, 129, 55-76, https://doi.org/10.1016/j.isprsjprs.2017.04.019, 2017.

Porter, C., Morin, P., Howat, I., Noh, M.-J., Bates, B., Peterman, K., Keesey, S., Schlenk, M., Gardiner, J., Tomko, K., Willis, M., Kelleher, C., Cloutier, M., Husby, E., Foga, S., Nakamura, H., Platson, M., Wethington, Michael, J., Williamson, C., Bauer, G., Enos, J., Arnold, G., Kramer, W., Becker, P., Doshi, A., D’Souza, C., Cummens, P., Laurier, F., and Bojesen, M.: ArcticDEM, https://doi.org/10.7910/DVN/OHHUKH, 2018.

Remy, F., Mazzega, P., Houry, S., Brossier, C., and Minster, J.: Mapping of the Topography of Continental Ice by Inversion of Satellitealtimeter Data, Journal of Glaciology, 35, 98-107, https://doi.org/10.3189/002214389793701419, 1989.

Roemer, S., Legrésy, B., Horwath, M., and Dietrich, R.: Refined analysis of radar altimetry data applied to the region of the subglacial Lake Vostok/Antarctica, Remote Sensing of Environment, 106, 269-284, https://doi.org/10.1016/j.rse.2006.02.026, 2007.

Schröder, L., Horwath, M., Dietrich, R., Helm, V., van den Broeke, M. R., and Ligtenberg, S. R. M.: Four decades of Antarctic surface elevation changes from multi-mission satellite altimetry, The Cryosphere, 13, 427-449, https://doi.org/10.5194/tc-13-427-2019, 2019.

Simonsen, S. B. and Sørensen, L. S.: Implications of changing scattering properties on Greenland ice sheet volume change from Cryosat-2 altimetry, Remote Sensing of Environment, 190, 207-216, https://doi.org/10.1016/j.rse.2016.12.012, 2017.

Slater, T., Shepherd, A., McMillan, M., Muir, A., Gilbert, L., Hogg, A. E., Konrad, H., and Parrinello, T.: A new digital elevation model of Antarctica derived from CryoSat-2 altimetry, The Cryosphere, 12, 1551-1562, https://doi.org/10.5194/tc-12-1551-2018, 2018.

Smith, B., Fricker, H. A., Holschuh, N., Gardner, A. S., Adusumilli, S., Brunt, K. M., Csatho, B., Harbeck, K., Huth, A., Neumann, T., Nilsson, J., and Siegfried, M. R.: Land ice height-retrieval algorithm for NASA's ICESat-2 photon-counting laser altimeter, Remote Sensing of Environment, 233, 111352, https://doi.org/10.1016/j.rse.2019.111352, 2019.

Smith, B., Fricker, H. A., Gardner, A., Siegfried, M. R., Adusumilli, S., Csathó, B. M., Holschuh, N., Nilsson, J., Paolo, F. S., and the ICESat-2 Science Team: ATLAS/ICESat-2 L3A Land Ice Height, Version 3, https://doi.org/10.5067/ATLAS/ATL06.003, [Date Accessed 2020-09-01], 2020a.

Smith, B., Hancock, D., Harbeck, K., Roberts, L., Neumann, T., Brunt, K., Fricker, H., Gardner, A., Siegfried, M., Adusumilli, S., Csathó, B., Holschuh, N., Nilsson, J., and Paolo, F.: Algorithm Theoretical Basis Document (ATBD) for Land Ice Along-Track Height Product (ATL06), Tech. rep., ICESat-2 Project Science Office, https://nsidc.org/sites/nsidc.org/files/technical-references/ICESat2_ATL06_ATBD_ r003.pdf, 2020b.

Wingham, D., Rapley, C., and D, G.: New Techniques in Satellite Altimeter Tracking Systems, in: Digest - International Geoscience and Remote Sensing Symposium (IGARSS), vol. ESA SP-254, pp. 1339-1344, Zurich, 1986. 